\title{
On Some New Fixed Point Results with Applications to Matrix Difference Equations
}

\author{
Marwan Amin Kutbi, ${ }^{1}$ Jamshaid Ahmad (D, ${ }^{2}$ and Muhammad Imran Shahzad ${ }^{3}$ \\ ${ }^{1}$ Department of Mathematics, King Abdulaziz University, P. O. Box 80203, Jeddah 21589, Saudi Arabia \\ ${ }^{2}$ Department of Mathematics, University of Jeddah, P. O. Box 80327, Jeddah 21589, Saudi Arabia \\ ${ }^{3}$ Department of Mathematics, Institute of Southern Punjab, Multan, Pakistan
}

Correspondence should be addressed to Jamshaid Ahmad; jkhan@uj.edu.sa

Received 17 February 2021; Accepted 7 May 2021; Published 1 June 2021

Academic Editor: Huseyin Isik

Copyright ( $) 2021$ Marwan Amin Kutbi et al. This is an open access article distributed under the Creative Commons Attribution License, which permits unrestricted use, distribution, and reproduction in any medium, provided the original work is properly cited.

The aim of this article is to discuss the convergence of iterative sequences of the Prešic type involving new classes of operators satisfying Prešić type $\Theta$-contractive condition in the context of metric spaces. Some examples are also provided to show the significance of the investigation of finding fixed points. Some convergence results for a class of matrix difference equations will be derived as application.

\section{Introduction and Preliminaries}

Banach's contraction principle [1] is one of the decisive results of fixed point theory. It states that if we have a self mapping $\mathscr{L}$ on a complete metric space $(\mathcal{S}, \sigma)$ and a constant $\lambda \in(0,1)$ such that

$$
\sigma(\mathscr{L} \mathfrak{a}, \mathscr{L} \mathfrak{b}) \leq \lambda \sigma(\mathfrak{a}, \mathfrak{b}),
$$

holds, $\forall \mathfrak{a}, \mathfrak{b} \in \mathcal{S}$, then there exists a unique $\mathfrak{a}^{*} \in \mathcal{S}$ such that $\mathfrak{a}^{*}=\mathscr{L} \mathfrak{a}^{*}$.

Because of its substance and accessibility, many authors have established various fascinating supplements and extensions of this principle (see [1-21] and references therein). From now to onward, we will consider $k$ as positive integer and $(\mathcal{S}, \sigma)$ as complete metric space.

In 1965, Prešić [2] generalized the famous Banach contraction principle and applied the obtained results to secure the convergence of a specific type of sequences. Prešić established the following theorem.

Theorem 1 (see [2]). Let $\mathscr{L}: \mathcal{S}^{k} \longrightarrow \mathcal{S}$ be a mapping satisfying the following contractive condition:

$$
\begin{aligned}
& \sigma\left(\mathscr{L}\left(\mathfrak{a}_{1}, \mathfrak{a}_{2}, \ldots, \mathfrak{a}_{k}\right), \mathscr{L}\left(\mathfrak{a}_{2}, \ldots, \mathfrak{a}_{k}, \mathfrak{a}_{k+1}\right)\right) \\
& \leq \lambda_{1} \sigma\left(\mathfrak{a}_{1}, \mathfrak{a}_{2}\right)+\lambda_{2} \sigma\left(\mathfrak{a}_{2}, \mathfrak{a}_{3}\right)+\cdots+\lambda_{k} \sigma\left(\mathfrak{a}_{k}, \mathfrak{a}_{k+1}\right),
\end{aligned}
$$

for every $\mathfrak{a}_{1}, \ldots, \mathfrak{a}_{k+1} \in \mathcal{S}$, where $\lambda_{1}, \lambda_{2}, \ldots, \lambda_{k}$ are nonnegative constants such that $\lambda_{1}+\lambda_{2}+\cdots+\lambda_{k}<1$. Then, $\forall \mathfrak{a}^{*} \in \mathcal{S}$ such that $\mathscr{L}\left(\mathfrak{a}^{*}, \ldots, \mathfrak{a}^{*}\right)=\mathfrak{a}^{*}$ and it is unique. Moreover if $\mathfrak{a}_{1}, \ldots, \mathfrak{a}_{k}$ are arbitrary points in $\mathcal{S}$ and for $n \in \mathbb{N}$,

$$
\mathfrak{a}_{n+k}=\mathscr{L}\left(\mathfrak{a}_{n}, \mathfrak{a}_{n+1}, \ldots, \mathfrak{a}_{n+k-1}\right)
$$

then the sequence $\left\{\mathfrak{a}_{n}\right\}$ is convergent and $\mathfrak{a}^{*}=\lim \mathfrak{a}_{n}=$ $\mathscr{L}\left(\operatorname{lima} \mathfrak{a}_{n}, \operatorname{lima}_{n}, \ldots, \operatorname{lima} \mathfrak{a}_{n}\right)$.

A mapping $\mathscr{L}: \mathcal{S}^{k} \longrightarrow \mathcal{S}$ satisfying inequality (2) is said to be a Prešić operator. A point $\mathfrak{a}^{*} \in \mathcal{S}$ is called a fixed point of $\mathscr{L}$ if $\mathfrak{a}^{*}=\mathscr{L}\left(\mathfrak{a}^{*}, \ldots, \mathfrak{a}^{*}\right)$. If $k=1$ in Theorem 1 , then we get the Banach contraction principle as a specific case.

Ćirić et al. [3] established the following theorem.

Theorem 2 (see [3]). Assume that $\mathscr{L}: \mathcal{\delta}^{k} \longrightarrow \mathcal{\delta}$ satisfies

$$
\begin{aligned}
& \sigma\left(\mathscr{L}\left(\mathfrak{a}_{1}, \mathfrak{a}_{2}, \ldots, \mathfrak{a}_{k}\right), \mathscr{L}\left(\mathfrak{a}_{2}, \ldots, \mathfrak{a}_{k}, \mathfrak{a}_{k+1}\right)\right) \\
& \leq \lambda \max \left\{\sigma\left(\mathfrak{a}_{1}, \mathfrak{a}_{2}\right), \sigma\left(\mathfrak{a}_{2}, \mathfrak{a}_{3}\right), \ldots, \sigma\left(\mathfrak{a}_{k}, \mathfrak{a}_{k+1}\right)\right\},
\end{aligned}
$$


for any $\mathfrak{a}_{1}, \ldots, \mathfrak{a}_{k+1} \in \mathcal{S}$, where $0<\lambda<1$. Then, $\exists \mathfrak{a}^{*} \in \mathcal{S}$ such that $\mathscr{L}\left(\mathfrak{a}^{*}, \ldots, \mathfrak{a}^{*}\right)=\mathfrak{a}^{*}$. Moreover, for any arbitrary points $\mathfrak{a}_{1}, \ldots, \mathfrak{a}_{k} \in \mathcal{S}$, the sequence given in (3) is convergent and

$$
\lim _{n \longrightarrow \infty} \mathfrak{a}_{n}=\mathscr{L}\left(\lim _{n \longrightarrow \infty} \mathfrak{a}_{n}, \ldots, \lim _{n \longrightarrow \infty} \mathfrak{a}_{n}\right) .
$$

If in addition,

$$
\sigma\left(\mathscr{L}\left(\mathfrak{a}^{*}, \ldots, \mathfrak{a}^{*}\right), \mathscr{L}\left(\mathfrak{a}^{\prime}, \ldots, \mathfrak{a}^{\prime}\right)\right)<\sigma\left(\mathfrak{a}^{*}, \mathfrak{a}^{\prime}\right),
$$

holds for all $\mathfrak{a}^{*}, \mathfrak{a}^{\prime} \in \mathcal{S}$, with $\mathfrak{a}^{*} \neq \mathfrak{a}^{\prime}$, then the fixed point in $\mathcal{S}$ is unique.

Pwfâcurar [4] obtained a convergence theorem for Prešić-Kannan contraction in this way.

Definition 1 (see [4]). Assume that $\mathscr{L}: \mathcal{S}^{k} \longrightarrow \mathcal{S}$. If $\exists \lambda \in \mathbb{R}$ with $0<\lambda k(k+1)<1$ such that

$$
\begin{aligned}
& \sigma\left(\mathscr{L}\left(\mathfrak{a}_{1}, \ldots, \mathfrak{a}_{k}\right), \mathscr{L}\left(\mathfrak{a}_{2}, \ldots, \mathfrak{a}_{k+1}\right)\right) \\
& \leq \lambda \sum_{i=1}^{k+1} \sigma\left(\mathfrak{a}_{i}, \mathscr{L}\left(\mathfrak{a}_{i}, \ldots, \mathfrak{a}_{i}\right)\right),
\end{aligned}
$$

holds for all $\left(\mathfrak{a}_{1}, \ldots, \mathfrak{a}_{k+1}\right) \in \mathcal{S}^{k+1}$. Then,

(i) $\mathscr{L}$ has a unique fixed point $\mathfrak{a}^{*} \in \mathcal{S}$.

(ii) For any arbitrary points $\mathfrak{a}_{1}, \ldots, \mathfrak{a}_{k} \in \mathcal{S}$, the sequence $\left\{\mathfrak{a}_{n}\right\}$ given by (3) converges to $\mathfrak{a}^{*}$. Supplementary in this direction, we refer the readers to $[5-8,10]$.

Very recently, Jleli et al. [11] gave a new variety of contraction called $\Theta$-contraction and established generalized results for these contractions.

Definition 2. Let $\Theta:(0, \infty) \longrightarrow(1, \infty)$ be a mapping satisfying the following:

(i) $\left(\Theta_{1}\right) \Theta$ is nondecreasing.

(ii) $\left(\Theta_{2}\right) \quad \forall\left\{t_{n}\right\} \subseteq R^{+}, \lim _{n \longrightarrow \infty} \Theta\left(t_{n}\right)=1 \Longleftrightarrow \lim _{n \longrightarrow \infty}$ $\left(t_{n}\right)=0$.

(iii) $\left(\Theta_{3}\right) \exists 0<h<1$ and $l \in(0, \infty]$ such that $\lim _{t \rightarrow 0^{+}}$ $\left((\Theta(t)-1) / t^{h}\right)=l$.

A mapping $\mathscr{L}: \mathcal{S} \longrightarrow \mathcal{S}$ is called a $\Theta$-contraction if there exist some $\lambda \in(0,1)$ and a function $\Theta$ satisfying $\left(\Theta_{1}\right)-\left(\Theta_{3}\right)$ such that

$$
\mathscr{L} \mathfrak{a} \neq \mathscr{L} \mathfrak{b} \Longrightarrow \Theta(\sigma(\mathscr{L} \mathfrak{a}, \mathscr{L} \mathfrak{b})) \leq[\Theta(\sigma(\mathfrak{a}, \mathfrak{b}))]^{\lambda},
$$

$\forall \mathfrak{a}, \mathfrak{b} \in \mathcal{S}$.

Theorem 3 (see [11]). Let $\mathscr{L}: \mathcal{S} \longrightarrow \mathcal{S}$ be a $\Theta$-contraction; then, $\mathscr{L}$ has a unique fixed point.

We represent by the $\Omega$ set of all mappings $\Theta:(0, \infty) \longrightarrow(1, \infty)$ satisfying $\left(\Theta_{1}\right)-\left(\Theta_{3}\right)$ consistent with Samet et al. [11] . For more details, we refer the following $[12,14-16,18-20]$ to the readers.

In this article, we discuss the convergence of $\left\{\mathfrak{a}_{n}\right\}$ given by (3), where $\mathscr{L}: \mathcal{S}^{k} \longrightarrow \mathcal{S}$ is a Prešić type $\Theta$-contraction. The given results unify and generalize various existing results of the literature.

\section{Main Results}

Motivated by the work of Samet et al. [11], we give the following definition.

Definition 3. A mapping $\mathscr{L}: \mathcal{S}^{k} \longrightarrow \mathcal{S}$ is called a Prešić type $\Theta$-contraction if there exists some $\lambda \in(0,1)$ such that

$$
\begin{aligned}
& \Theta\left(\sigma\left(\mathscr{L}\left(\mathfrak{a}_{1}, \ldots, \mathfrak{a}_{k}\right), \mathscr{L}\left(\mathfrak{a}_{2}, \ldots, \mathfrak{a}_{k+1}\right)\right)\right) \\
& \leq\left[\Theta\left(\max \left\{\sigma\left(\mathfrak{a}_{i}, \mathfrak{a}_{i+1}\right): \quad 1 \leq i \leq k\right\}\right)\right]^{\lambda},
\end{aligned}
$$

$\forall\left(\mathfrak{a}_{1}, \ldots, \mathfrak{a}_{k+1}\right) \in \mathcal{S}^{k+1}$ with $\mathscr{L}\left(\mathfrak{a}_{1}, \ldots, \mathfrak{a}_{k}\right) \neq \mathscr{L} \quad\left(\mathfrak{a}_{2}, \ldots\right.$, $\left.\mathfrak{a}_{k+1}\right)$.

Note that for $\Theta(t)=e^{\sqrt{t}}$, Prešić type $\Theta$-contractive condition is reduced to

$$
\begin{array}{r}
\sigma\left(\mathscr{L}\left(\mathfrak{a}_{1}, \ldots, \mathfrak{a}_{k}\right), \mathscr{L}\left(\mathfrak{a}_{2}, \ldots, \mathfrak{a}_{k+1}\right)\right) \\
\leq \lambda^{2} \max \left\{\sigma\left(\mathfrak{a}_{i}, \mathfrak{a}_{i+1}\right): \quad 1 \leq i \leq k\right\},
\end{array}
$$

$\forall\left(\mathfrak{a}_{1}, \ldots, \mathfrak{a}_{k+1}\right) \in \mathcal{S}^{k+1}, \mathscr{L}\left(\mathfrak{a}_{1}, \ldots, \mathfrak{a}_{k}\right) \neq \mathscr{L}\left(\mathfrak{a}_{2}, \ldots, \mathfrak{a}_{k+1}\right)$.

Furthermore, for $\left(\mathfrak{a}_{1}, \ldots, \mathfrak{a}_{k+1}\right) \in \delta^{k+1}$ such that $\mathscr{L}\left(\mathfrak{a}_{1}, \ldots, \mathfrak{a}_{k}\right)=\mathscr{L}\left(\mathfrak{a}_{2}, \ldots, \mathfrak{a}_{k+1}\right)$, inequality (4) is also satisfied, i.e., $\mathscr{L}$ is a Ćirić-Prešić contraction.

Remark 1. Every Prešić type $\Theta$-contraction $\mathscr{L}$ is a Prešić operator by $\left(\Theta_{1}\right)$ and $(7)$, that is,

$$
\begin{aligned}
& \sigma\left(\mathscr{L}\left(\mathfrak{a}_{1}, \ldots, \mathfrak{a}_{k}\right), \mathscr{L}\left(\mathfrak{a}_{2}, \ldots, \mathfrak{a}_{k+1}\right)\right) \\
& <\max \left\{\sigma\left(\mathfrak{a}_{i}, \mathfrak{a}_{i+1}\right): \quad 1 \leq i \leq k\right\},
\end{aligned}
$$

$\forall\left(\mathfrak{a}_{1}, \ldots, \mathfrak{a}_{k+1}\right) \in \mathcal{S}^{k+1}, \quad \mathscr{L}\left(\mathfrak{a}_{1}, \ldots, \mathfrak{a}_{k}\right) \neq \mathscr{L}\left(\mathfrak{a}_{2}, \ldots, \mathfrak{a}_{k+1}\right)$. Thus, each Prešić type $\Theta$-contraction $\mathscr{L}$ is a continuous function.

Now we present our main result in this way.

Theorem 4. Assume that $\mathscr{L}: \mathcal{S}^{k} \longrightarrow \mathcal{S}$ a Prešić type $\Theta$-contraction. Then, for any arbitrary points $\mathfrak{a}_{1}, \ldots, \mathfrak{a}_{k} \in \mathcal{S}$, the sequence $\left\{\mathfrak{a}_{n}\right\}$ given by (3) converges to $\mathfrak{a}^{*}$ in $\mathcal{S}$ and $\mathfrak{a}^{*}$ is a fixed point of $\mathscr{L}$. Additionally, if $\sigma(\mathscr{L}(\mathfrak{a}, \ldots, \mathfrak{a})$ $\neq \mathscr{L}(\mathfrak{b}, \ldots, \mathfrak{b})$ implies that

$$
\Theta(\sigma(\mathscr{L}(\mathfrak{a}, \ldots, \mathfrak{a}), \mathscr{L}(\mathfrak{b}, \ldots, \mathfrak{b}))) \leq[\sigma(\mathfrak{a}, \mathfrak{b})]^{\lambda},
$$

$\forall \mathfrak{a}, \mathfrak{b} \in \mathcal{S}$ with $\mathfrak{a} \neq \mathfrak{b}$, then there exists unique $\mathfrak{a}^{*} \in \mathcal{S}$ such that $\mathscr{L} \mathfrak{a}^{*}=\mathfrak{a}^{*}$.

Proof. Let $\mathfrak{a}_{1}, \ldots, \mathfrak{a}_{k}$ be arbitrary $k$ elements in $\mathcal{S}$. Define $\left\{\mathfrak{a}_{n}\right\}$ in $\mathcal{S}$ by

$$
\mathfrak{a}_{n+k}=\mathscr{L}\left(\mathfrak{a}_{n}, \mathfrak{a}_{n+1}, \ldots, \mathfrak{a}_{n+k-1}\right),
$$

for $n \in \mathbb{N}$. If for some $n_{0} \in\{1,2,3, \ldots, k\}$, we have $\mathfrak{a}_{n_{0}}=\mathfrak{a}_{n_{0}+1}$, then

$$
\begin{aligned}
\mathfrak{a}_{n_{0}+k} & =\mathscr{L}\left(\mathfrak{a}_{n_{0}}, \mathfrak{a}_{n_{0}+1}, \ldots, \mathfrak{a}_{n_{0}+k-1}\right) \\
& =\mathscr{L}\left(\mathfrak{a}_{n_{0}+k}, \mathfrak{a}_{n_{0}+k}, \ldots, \mathfrak{a}_{n_{0}+k}\right),
\end{aligned}
$$

that is, $\mathfrak{a}_{n_{0}+k}$ is a fixed point of $\mathscr{L}$ and we have nothing to prove. So, we assume that $\mathfrak{a}_{n+k} \neq \mathfrak{a}_{n+k+1}$ for all $n \in \mathbb{N}$. Represent $\beta_{n+k}=\sigma\left(\mathfrak{a}_{n+k}, \mathfrak{a}_{n+k+1}\right) \quad$ for $n \in \mathbb{N}$ and 
$\mu=\max \left\{\sigma\left(\mathfrak{a}_{1}, \mathfrak{a}_{2}\right), \sigma\left(\mathfrak{a}_{2}, \mathfrak{a}_{3}\right), \ldots, \sigma\left(\mathfrak{a}_{k}, \mathfrak{a}_{k+1}\right)\right\} ;$ then, we obtain $\beta_{n+k}>0, \forall n \in \mathbb{N}$, and $\mu>0$. Thus, for $n \leq k$, we get

$$
\begin{aligned}
1<\Theta\left(\beta_{k+1}\right) & =\Theta\left(\sigma\left(\mathfrak{a}_{k+1}, \mathfrak{a}_{k+2}\right)\right) \\
& =\Theta\left(\sigma\left(\mathscr{L}\left(\mathfrak{a}_{1}, \mathfrak{a}_{2}, \ldots, \mathfrak{a}_{k}\right), \mathscr{L}\left(\mathfrak{a}_{2}, \mathfrak{a}_{3}, \ldots, \mathfrak{a}_{k+1}\right)\right)\right) \\
& \leq\left[\Theta\left(\max \left\{\sigma\left(\mathfrak{a}_{i}, \mathfrak{a}_{i+1}\right): \quad 1 \leq i \leq k\right\}\right)\right]^{\lambda} \\
& =[\Theta(\mu)]^{\lambda}, \\
1 & <\Theta\left(\beta_{k+2}\right)=\Theta\left(\sigma\left(\mathfrak{a}_{k+2}, \mathfrak{a}_{k+3}\right)\right) \\
& =\Theta\left(\sigma\left(\mathscr{L}\left(\mathfrak{a}_{2}, \mathfrak{a}_{3}, \ldots, \mathfrak{a}_{k+1}\right), \mathscr{L}\left(\mathfrak{a}_{3}, \mathfrak{a}_{4}, \ldots, \mathfrak{a}_{k+2}\right)\right)\right) \\
& \leq\left[\Theta\left(\max \left\{\sigma\left(\mathfrak{a}_{i}, \mathfrak{a}_{i+1}\right): \quad 2 \leq i \leq k+1\right\}\right)\right]^{\lambda} \\
& \leq[\Theta(\mu)]^{\lambda^{2}} \\
& \vdots,
\end{aligned}
$$

and so on. Hence,

$$
\begin{aligned}
1<\Theta\left(\beta_{k+n}\right) & =\Theta\left(\sigma\left(\mathfrak{a}_{n+k}, \mathfrak{a}_{n+k+1}\right)\right) \\
& =\Theta\left(\sigma\left(\mathscr{L}\left(\mathfrak{a}_{n}, \mathfrak{a}_{n+1}, \ldots, \mathfrak{a}_{n+k-1}\right), \mathscr{L}\left(\mathfrak{a}_{n+1}, \mathfrak{a}_{n+2}, \ldots, \mathfrak{a}_{n+k}\right)\right)\right) \\
& \leq[\Theta(\mu)]^{\lambda^{n}}
\end{aligned}
$$

for $n \geq 1$. Letting $n \longrightarrow \infty$, we get by $\left(\Theta_{2}\right)$ that

$$
\lim _{n \longrightarrow \infty} \Theta\left(\beta_{k+n}\right)=1 \Longleftrightarrow \lim _{n \longrightarrow \infty} \beta_{k+n}=0 .
$$

By $\left(\Theta_{3}\right), \exists 0<h<1$ and $l \in(0, \infty]$ such that

$$
\lim _{n \rightarrow \infty} \frac{\Theta\left(\beta_{k+n}\right)-1}{\beta_{k+n}^{h}}=l .
$$

Let $l<\infty$ and $\omega=(l / 2)>0$. By definition of the limit, $\exists n_{1} \in \mathbb{N}$ such that

$$
\left|\frac{\Theta\left(\beta_{k+n}\right)-1}{\beta_{k+n}^{h}}-l\right| \leq \omega,
$$

$\forall n>n_{1}$. It shows that

$$
\frac{\Theta\left(\beta_{k+n}\right)-1}{\beta_{k+n}^{h}} \geq l-\omega=\frac{l}{2}=\omega,
$$

$\forall n>n_{1}$. Then,

$$
n \beta_{k+n}^{h} \leq \bowtie n\left[\Theta\left(\beta_{k+n}\right)-1\right]
$$

$\forall n>n_{1}$, where $\Phi=(1 / \omega)$. Now we assume that $l=\infty$. Let $\omega>0$. By definition of the limit, $\exists n_{1} \in \mathbb{N}$ such that

$$
\omega \leq \frac{\Theta\left(\beta_{k+n}\right)-1}{\beta_{k+n}^{h}},
$$

$\forall n>n_{1}$. This implies that

$$
n \beta_{k+n}^{h} \leq \bowtie n\left[\Theta\left(\beta_{k+n}\right)-1\right]
$$

$\forall n>n_{1}$, where $\Phi=(1 / \omega)$. Thus, in all cases, there exist $\Phi>0$ and $n_{1} \in \mathbb{N}$ such that

$$
n \beta_{k+n}^{h} \leq \bowtie n\left[\Theta\left(\beta_{k+n}\right)-1\right],
$$

for all $n>n_{1}$. Thus, by (10), we get

$$
n \beta_{k+n}^{h} \leq \bowtie n\left([\Theta(\mu)]^{\lambda^{n}}-1\right) .
$$

Letting $n \longrightarrow \infty$, we get

$$
\lim _{n \longrightarrow \infty} n \beta_{k+n}^{h}=0 \text {. }
$$

Thus, $\exists n_{2} \in \mathbb{N}$ such that

$$
n \beta_{k+n}^{h} \leq 1,
$$

$\forall n>n_{2}$. Therefore, we get

$$
\beta_{k+n} \leq \frac{1}{n^{1 / h}}
$$

for all $n>n_{2}$. Now we show that $\left\{\mathfrak{a}_{n}\right\}$ is a Cauchy sequence. For $m>n>n_{2}$, we have

$$
\begin{aligned}
\sigma\left(\mathfrak{a}_{k+n}, \mathfrak{a}_{k+m}\right)= & \sigma\left(\mathscr{L}\left(\mathfrak{a}_{n}, \ldots, \mathfrak{a}_{k+n-1}\right), \mathscr{L}\left(\mathfrak{a}_{m}, \ldots, \mathfrak{a}_{k+m-1}\right)\right) \\
\leq & \sigma\left(\mathscr{L}\left(\mathfrak{a}_{n}, \ldots, \mathfrak{a}_{k+n-1}\right), \mathscr{L}\left(\mathfrak{a}_{n+1}, \ldots, \mathfrak{a}_{k+n}\right)\right) \\
& +\sigma\left(\mathscr{L}\left(\mathfrak{a}_{n+1}, \ldots, \mathfrak{a}_{k+n}\right), \mathscr{L}\left(\mathfrak{a}_{n+2}, \ldots, \mathfrak{a}_{k+n+1}\right)\right) \\
& +\cdots+\sigma\left(\mathscr{L}\left(\mathfrak{a}_{m-1}, \ldots, \mathfrak{a}_{k+m-2}\right), \mathscr{L}\left(\mathfrak{a}_{m}, \ldots, \mathfrak{a}_{k+m-1}\right)\right) \\
= & \sigma\left(\mathfrak{a}_{n+k}, \mathfrak{a}_{n+k+1}\right)+\sigma\left(\mathfrak{a}_{n+k+1}, \mathfrak{a}_{n+k+2}\right)+\cdots+\sigma\left(\mathfrak{a}_{m+k-1}, \mathfrak{a}_{m+k}\right) \\
= & \beta_{n+k}+\beta_{n+k+1}+\cdots+\beta_{m+k-1} \\
= & \sum_{i=n}^{m-1} \beta_{i+k}<\sum_{i=n}^{\infty} \beta_{i+k} \leq \sum_{i=n}^{\infty} \frac{1}{i^{1 / h}}<\infty .
\end{aligned}
$$


This proves that $\left\{\mathfrak{a}_{n}\right\}$ is Cauchy in $(\mathcal{S}, \sigma)$. As $(\mathcal{S}, \sigma)$ is complete, $\exists \mathfrak{a}^{*}$ in $\mathcal{S}$ such that

$$
\lim _{n, m \longrightarrow \infty} \sigma\left(\mathfrak{a}_{n}, \mathfrak{a}_{m}\right)=\lim _{n \longrightarrow \infty} \sigma\left(\mathfrak{a}_{n}, \mathfrak{a}^{*}\right)=0 .
$$

Now as $\mathscr{L}$ is continuous, we get

$$
\begin{aligned}
\mathfrak{a}^{*} & =\lim _{n \longrightarrow \infty} \mathfrak{a}_{n+k}=\lim _{n \longrightarrow \infty} \mathscr{L}\left(\mathfrak{a}_{n}, \mathfrak{a}_{n+1}, \ldots, \mathfrak{a}_{n+k-1}\right) \\
& =\mathscr{L}\left(\lim _{n \longrightarrow \infty} \mathfrak{a}_{n}, \lim _{n \longrightarrow \infty} \mathfrak{a}_{n+1}, \ldots, \lim _{n \longrightarrow \infty} \mathfrak{a}_{n+k-1}\right) \\
& =\mathscr{L}\left(\mathfrak{a}^{*}, \mathfrak{a}^{*}, \ldots, \mathfrak{a}^{*}\right) .
\end{aligned}
$$

Now we show the uniqueness of fixed point of mapping $\mathscr{L}$. We suppose on the contrary that $\exists \mathfrak{a}^{*}, \mathfrak{a}^{\prime} \in \mathcal{S}$ so that $\mathfrak{a}^{*}=$ $\mathscr{L}\left(\mathfrak{a}^{*}, \mathfrak{a}^{*}, \ldots, \mathfrak{a}^{*}\right)$ and $\mathfrak{a}^{\prime}=\mathscr{L}\left(\mathfrak{a}^{\prime}, \mathfrak{a}^{\prime}, \ldots, \mathfrak{a}^{\prime}\right)$ with $\mathfrak{a}^{*} \neq \mathfrak{a}^{\prime}$. Thus, $\mathscr{L}\left(\mathfrak{a}^{*}, \mathfrak{a}^{*}, \ldots, \mathfrak{a}^{*}\right) \neq \mathscr{L}\left(\mathfrak{a}^{\prime}, \mathfrak{a}^{\prime}, \ldots, \mathfrak{a}^{\prime}\right)$. Hence, by given assumption, we have

$$
\begin{aligned}
\Theta\left(\sigma\left(\mathfrak{a}^{*}, \mathfrak{a}^{\prime}\right)\right) & =\Theta\left(\sigma\left(\mathscr{L}\left(\mathfrak{a}^{*}, \mathfrak{a}^{*}, \ldots, \mathfrak{a}^{*}\right), \mathscr{L}\left(\mathfrak{a}^{\prime}, \mathfrak{a}^{\prime}, \ldots, \mathfrak{a}^{\prime}\right)\right)\right) \\
& \leq\left[\Theta\left(\sigma\left(\mathfrak{a}^{*}, \mathfrak{a}^{\prime}\right)\right]^{\lambda},\right.
\end{aligned}
$$

a contradiction as $\lambda \in(0,1)$. Therefore, $\mathfrak{a}^{*}=\mathfrak{a}^{\prime}$.

Example 1. Consider the sequence $\left\{\mathfrak{a}_{n}\right\}$ as follows:

$$
\begin{aligned}
\mathfrak{a}_{1} & =1 \\
\mathfrak{a}_{2} & =1+5 \\
& \vdots \\
\mathfrak{a}_{n} & =1+5+9+\cdots+(4 n-3)=n(2 n-1) .
\end{aligned}
$$

Let $\mathcal{S}=\left\{\mathfrak{a}_{n}: n \in \mathbb{N}\right\}$ and $\sigma\left(\mathfrak{a}^{*}, \mathfrak{a}^{\prime}\right)=\left|\mathfrak{a}^{*}-\mathfrak{a}^{\prime}\right|$. Then, $(\mathcal{S}$, $\sigma)$ becomes a complete metric space. Consider $\mathscr{L}: \mathcal{S}^{2} \longrightarrow \mathcal{S}$ by

$$
\mathscr{L}\left(\mathfrak{a}_{n}^{*}, \mathfrak{a}_{n}^{\prime}\right)= \begin{cases}\frac{\mathfrak{a}_{n-1}^{*}+\mathfrak{a}_{n-1}^{\prime}}{2}, & \text { for } n>1, \\ \frac{\mathfrak{a}_{1}^{*}+\mathfrak{a}_{1}^{\prime}}{2}, & \text { otherwise. }\end{cases}
$$

For $n>3$, we get

$$
\begin{aligned}
& \sigma\left(\mathscr{L}\left(\mathfrak{a}_{n-2}, \mathfrak{a}_{n-1}\right), \mathscr{L}\left(\mathfrak{a}_{n-1}, \mathfrak{a}_{n}\right)\right) \\
= & \sigma\left(\frac{\mathfrak{a}_{n-3}+\mathfrak{a}_{n-2}}{2}, \frac{\mathfrak{a}_{n-2}+\mathfrak{a}_{n-1}}{2}\right) \\
= & \frac{1}{2}|((n-3)(2 n-7)+(n-2)(2 n-5))-((n-2)(2 n-5)+(n-1)(2 n-3))| \\
= & \frac{1}{2}\left|2 n^{2}-13 n+21+2 n^{2}-9 n+10-\left(2 n^{2}-9 n+10+2 n^{2}-5 n+3\right)\right| \\
= & \frac{1}{2}(8 n-18)=4 n-9, \\
& \max \left\{\sigma\left(\mathfrak{a}_{n-2}, \mathfrak{a}_{n-1}\right), \sigma\left(\mathfrak{a}_{n-1}, \mathfrak{a}_{n}\right)\right\} \\
= & \max \{|(n-2)(2 n-5)-(n-1)(2 n-3)|,|(n-1)(2 n-3)-n(2 n-1)|\} \\
& \max \{4 n-7,4 n-3\}=4 n-3 .
\end{aligned}
$$

Now

$$
\begin{aligned}
& \lim _{n \longrightarrow \infty} \frac{\sigma\left(\mathscr{L}\left(\mathfrak{a}_{n-2}, \mathfrak{a}_{n-1}\right), \mathscr{L}\left(\mathfrak{a}_{n-1}, \mathfrak{a}_{n}\right)\right)}{\max \left\{\sigma\left(\mathfrak{a}_{n-2}, \mathfrak{a}_{n-1}\right), \sigma\left(\mathfrak{a}_{n-1}, \mathfrak{a}_{n}\right)\right\}} \\
& =\lim _{n \longrightarrow \infty} \frac{4 n-9}{4 n-3}=1 .
\end{aligned}
$$

Thus,

$$
\begin{aligned}
& \sigma\left(\mathscr{L}\left(\mathfrak{a}_{n-2}, \mathfrak{a}_{n-1}\right), \mathscr{L}\left(\mathfrak{a}_{n-1}, \mathfrak{a}_{n}\right)\right) \\
& \leq \lambda \max \left\{\sigma\left(\mathfrak{a}_{n-2}, \mathfrak{a}_{n-1}\right), \sigma\left(\mathfrak{a}_{n-1}, \mathfrak{a}_{n}\right)\right\}
\end{aligned}
$$

does not hold for $\lambda \in(0,1)$. Thus, the main hypothesis of Theorem 2 in [3] is not satisfied. Now, by considering the mapping $\Theta:(0, \infty) \longrightarrow(1, \infty)$ defined by

$$
\Theta(t)=e^{t e^{t}},
$$


we can easily show that $\Theta \in \Omega$ and $\mathscr{L}$ is Prešić type $\Theta$-contraction. Indeed, the following holds:

$$
\begin{aligned}
& e^{\sqrt{\sigma\left(\mathscr{L}\left(\mathfrak{a}_{i}, \mathfrak{a}_{i+1}\right)\right), \mathscr{L}\left(\mathfrak{a}_{i+1}, \mathfrak{a}_{i+2}\right) e^{\sigma\left(\mathscr{L}\left(\mathfrak{a}_{i}, a_{i+1}\right)\right), \mathscr{L}\left(\mathfrak{a}_{i+1}, a_{i+2}\right)}}} \\
& \leq e^{\lambda \sqrt{\sigma\left(\left(\mathfrak{a}_{i}, \mathfrak{a}_{i+1}\right)\right),\left(\mathfrak{a}_{i+1}, \mathfrak{a}_{i+2}\right) e^{\sigma\left(\left(a_{i}, a_{i+1}\right)\right),\left(a_{i+1}, \mathfrak{a}_{i+2}\right)}}}
\end{aligned}
$$

for $\mathscr{L}\left(\mathfrak{a}_{i}, \mathfrak{a}_{i+1}\right) \neq \mathscr{L}\left(\mathfrak{a}_{i+1}, \mathfrak{a}_{i+2}\right), i=1,2, \ldots$, and for some $\lambda \in(0,1)$. The above condition is equivalent to

$$
\begin{aligned}
& \sigma\left(\mathscr{L}\left(\mathfrak{a}_{i}, \mathfrak{a}_{i+1}\right)\right), \mathscr{L}\left(\mathfrak{a}_{i+1}, \mathfrak{a}_{i+2}\right) \\
& \cdot e^{\sigma\left(\mathscr{L}\left(\mathfrak{a}_{i}, \mathfrak{a}_{i+1}\right)\right), \mathscr{L}\left(\mathfrak{a}_{i+1}, \mathfrak{a}_{i+2}\right)} \\
& \leq \lambda^{2} \max \left\{\sigma\left(\left(\mathfrak{a}_{i}, \mathfrak{a}_{i+1}\right)\right), \sigma\left(\mathfrak{a}_{i+1}, \mathfrak{a}_{i+2}\right)\right\} \\
& \cdot e^{\max \left\{\sigma\left(\left(\mathfrak{a}_{i}, \mathfrak{a}_{i+1}\right)\right), \sigma\left(\mathfrak{a}_{i+1}, \mathfrak{a}_{i+2}\right)\right\}} .
\end{aligned}
$$

So, we have to check that

$$
\frac{\sigma\left(\mathscr{L}\left(\mathfrak{a}_{i}, \mathfrak{a}_{i+1}\right)\right), \mathscr{L}\left(\mathfrak{a}_{i+1}, \mathfrak{a}_{i+2}\right) \cdot e^{\sigma\left(\mathscr{L}\left(\mathfrak{a}_{i}, \mathfrak{a}_{i+1}\right)\right), \mathscr{L}\left(\mathfrak{a}_{i+1}, \mathfrak{a}_{i+2}\right)}}{\max \left\{\sigma\left(\left(\mathfrak{a}_{i}, \mathfrak{a}_{i+1}\right)\right), \sigma\left(\mathfrak{a}_{i+1}, \mathfrak{a}_{i+2}\right)\right\} \cdot e^{\max \left\{\sigma\left(\left(\mathfrak{a}_{i}, \mathfrak{a}_{i+1}\right)\right), \sigma\left(\mathfrak{a}_{i+1}, \mathfrak{a}_{i+2}\right)\right\}}}
$$$$
\leq \lambda^{2}
$$

for some $\lambda \in(0,1)$. We discuss these two cases.

Case 1. For $i=n=1$, we have

$$
\begin{aligned}
& \frac{\sigma\left(\mathscr{L}\left(\mathfrak{a}_{1}, \mathfrak{a}_{2}\right)\right), \mathscr{L}\left(\mathfrak{a}_{2}, \mathfrak{a}_{3}\right) e^{\sigma\left(\mathscr{L}\left(\mathfrak{a}_{1}, \mathfrak{a}_{2}\right)\right), \mathscr{L}\left(\mathfrak{a}_{2}, \mathfrak{a}_{3}\right)}}{\max \left\{\sigma\left(\left(\mathfrak{a}_{1}, \mathfrak{a}_{2}\right)\right), \sigma\left(\mathfrak{a}_{2}, \mathfrak{a}_{3}\right)\right\} e^{\max \left\{\sigma\left(\left(\mathfrak{a}_{1}, \mathfrak{a}_{1}\right)\right), \sigma\left(\mathfrak{a}_{2}, \mathfrak{a}_{3}\right)\right\}}} \\
= & \frac{\sigma\left(\left(\left(\mathfrak{a}_{1}+\mathfrak{a}_{1}\right) / 2\right),\left(\left(\mathfrak{a}_{1}+\mathfrak{a}_{2}\right) / 2\right)\right) e^{\sigma\left(\left(\left(\mathfrak{a}_{1}+\mathfrak{a}_{1}\right) / 2\right),\left(\left(\mathfrak{a}_{1}+\mathfrak{a}_{2}\right) / 2\right)\right)}}{\max \left\{\sigma\left(\left(\mathfrak{a}_{1}, \mathfrak{a}_{2}\right)\right), \sigma\left(\mathfrak{a}_{2}, \mathfrak{a}_{3}\right)\right\} e^{\max \left\{\sigma\left(\left(\mathfrak{a}_{1}, \mathfrak{a}_{2}\right)\right), \sigma\left(\mathfrak{a}_{2}, \mathfrak{a}_{3}\right)\right\}}} \\
= & \frac{\sigma(1,(7 / 2)) e^{\sigma(1,(7 / 2))}}{\max \{\sigma((1,6)), \sigma(6,15)\} e^{\max \{\sigma((1,6)), \sigma(6,15)\}}} \\
= & \frac{5}{18} e^{(-13 / 2)} \\
< & e^{-2}
\end{aligned}
$$

Case 2. For $i=n>1$, we have

$$
\begin{aligned}
& \frac{\sigma\left(\mathscr{L}\left(\mathfrak{a}_{n}, \mathfrak{a}_{n+1}\right)\right), \mathscr{L}\left(\mathfrak{a}_{n+1}, \mathfrak{a}_{n+2}\right) \cdot e^{\sigma\left(\mathscr{L}\left(\mathfrak{a}_{n}, \mathfrak{a}_{n+1}\right)\right), \mathscr{L}\left(\mathfrak{a}_{n+1}, \mathfrak{a}_{n+2}\right)}}{\max \left\{\sigma\left(\left(\mathfrak{a}_{n}, \mathfrak{a}_{n+1}\right)\right), \sigma\left(\mathfrak{a}_{n+1}, \mathfrak{a}_{n+2}\right)\right\} \cdot e^{\max \left\{\sigma\left(\left(\mathfrak{a}_{n}, \mathfrak{a}_{n+1}\right)\right), \sigma\left(\mathfrak{a}_{n+1}, \mathfrak{a}_{n+2}\right)\right\}}} \\
& =\frac{\sigma\left(\left(\left(\mathfrak{a}_{n-1}+\mathfrak{a}_{n}\right) / 2\right),\left(\left(\mathfrak{a}_{n}+\mathfrak{a}_{n+1}\right) / 2\right)\right) \cdot e^{\sigma\left(\left(\left(\mathfrak{a}_{n-1}+\mathfrak{a}_{n}\right) / 2\right),\left(\left(\mathfrak{a}_{n}+\mathfrak{a}_{n+1}\right) / 2\right)\right)}}{\max \left\{\sigma\left(\left(\mathfrak{a}_{n}, \mathfrak{a}_{n+1}\right)\right), \sigma\left(\mathfrak{a}_{n+1}, \mathfrak{a}_{n+2}\right)\right\} \cdot e^{\max \left\{\sigma\left(\left(\mathfrak{a}_{n}, \mathfrak{a}_{n+1}\right)\right), \sigma\left(\mathfrak{a}_{n+1}, \mathfrak{a}_{n+2}\right)\right\}}} \\
& =\frac{(1 / 2)\left\{\left(4 n^{2}-6 n+3\right)-\left(4 n^{2}+2 n+1\right)\right\} \cdot e^{(1 / 2)\left\{\left(4 n^{2}-6 n+3\right)-\left(4 n^{2}+2 n+1\right)\right\}}}{\max \{4 n+1,2 n+2\} \cdot e^{\max \{4 n+1,2 n+2\}}} \\
& =\frac{-4 n+1}{4 n+1} e^{-8 n} \\
& <e^{-2},
\end{aligned}
$$

with $\lambda=e^{-1}$.

Thus, all the conditions of Theorem 4 are satisfied and $(1,1)$ is the unique fixed point of $\mathscr{L}$.

Example 2. Let $\mathcal{S}=[0,1]$ and $\sigma$ be such that

$$
\sigma\left(\mathfrak{a}^{*}, \mathfrak{a}^{\prime}\right)=\left|\mathfrak{a}^{*}-\mathfrak{a}^{\prime}\right|,
$$

and $\mathscr{L}: \mathcal{S}^{k} \longrightarrow \mathcal{S}$ be defined by
$\mathscr{L}\left(\mathfrak{a}_{1}, \ldots, \mathfrak{a}_{k}\right)=\frac{\mathfrak{a}_{1}+\mathfrak{a}_{k}}{4 k}$ for all $\mathfrak{a}_{1}, \ldots, \mathfrak{a}_{k} \in \mathcal{S}$.

Define the mapping $\Theta:(0, \infty) \longrightarrow(1, \infty)$ given by

$$
\Theta(t)=e^{\sqrt{t}} \text {. }
$$

It is given in [11] that $\Theta \in \Omega$. Now for $\mathfrak{a}_{1}, \mathfrak{a}_{2}, \ldots, \mathfrak{a}_{k+1} \in \mathcal{S}$, we have

$$
\sigma\left(\mathscr{L}\left(\mathfrak{a}_{1}, \ldots, \mathfrak{a}_{k}\right), \mathscr{L}\left(\mathfrak{a}_{2}, \ldots, \mathfrak{a}_{k+1}\right)\right)>0 .
$$


We have

$$
\begin{aligned}
& \Theta\left(\sigma\left(\mathscr{L}\left(\mathfrak{a}_{1}, \ldots, \mathfrak{a}_{k}\right), \mathscr{L}\left(\mathfrak{a}_{2}, \ldots, \mathfrak{a}_{k+1}\right)\right)\right) \\
& =e^{\sqrt{\sigma\left(\mathscr{L}\left(\mathfrak{a}_{1}, \ldots, \mathfrak{a}_{k}\right), \mathscr{L}\left(\mathfrak{a}_{2}, \ldots, \mathfrak{a}_{k+1}\right)\right)}} \\
& =e^{\sqrt{(1 / 4 k)\left|\left(\mathfrak{a}_{1}-\mathfrak{a}_{2}\right)+\left(\mathfrak{a}_{k}-\mathfrak{a}_{k+1}\right)\right|}} \\
& =e^{(1 / 2 \sqrt{k}) \sqrt{\left|\left(\mathfrak{a}_{1}-\mathfrak{a}_{2}\right)+\left(\mathfrak{a}_{k}-\mathfrak{a}_{k+1}\right)\right|}} \\
& \leq e^{(1 / 2) \sqrt{\max \left\{\sigma\left(\mathfrak{a}_{1}, \mathfrak{a}_{2}\right), \sigma\left(\mathfrak{a}_{k}, \mathfrak{a}_{k+1}\right)\right\}}}
\end{aligned}
$$

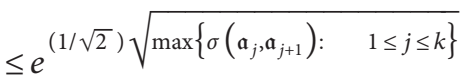

$$
\begin{aligned}
& =\left[\Theta\left(\max \left\{\sigma\left(\mathfrak{a}_{j}, \mathfrak{a}_{j+1}\right): \quad 1 \leq j \leq k\right\}\right)\right]^{\lambda} .
\end{aligned}
$$

with $\lambda=1 / \sqrt{2}$. Moreover, for all $\mathfrak{a}^{*}, \mathfrak{a}^{\prime} \in \mathcal{S}$ with $\mathfrak{a}^{*} \neq \mathfrak{a}^{\prime}$, we have

$$
\begin{aligned}
& \sigma\left(\mathscr{L}\left(\mathfrak{a}^{*}, \mathfrak{a}^{*}, \ldots, \mathfrak{a}^{*}\right), \mathscr{L}\left(\mathfrak{a}^{\prime}, \mathfrak{a}^{\prime} \ldots, \mathfrak{a}^{\prime}\right)\right) \\
= & \frac{\left|\mathfrak{a}^{*}-\mathfrak{a}^{\prime}\right|}{2 k}>0, \\
& \Theta\left(\sigma\left(\mathscr{L}\left(\mathfrak{a}^{*}, \mathfrak{a}^{*}, \ldots, \mathfrak{a}^{*}\right), \mathscr{L}\left(\mathfrak{a}^{\prime}, \mathfrak{a}^{\prime} \ldots, \mathfrak{a}^{\prime}\right)\right)\right) \\
= & \Theta\left(\frac{\left|\mathfrak{a}^{*}-\mathfrak{a}^{\prime}\right|}{2 k}\right) \\
= & e^{\sqrt{\left(\left|\mathfrak{a}^{*}-\mathfrak{a}^{\prime}\right| / 2 k\right)}}=e^{(1 / \sqrt{2 k}) \sqrt{\left|\mathfrak{a}^{*}-\mathfrak{a}^{\prime}\right|}} \\
\leq & e^{(1 / \sqrt{2}) \sqrt{\left|\mathfrak{a}^{*}-\mathfrak{a}^{\prime}\right|}} \\
= & {\left[\Theta\left(\sigma\left(\mathfrak{a}^{*}, \mathfrak{a}^{\prime}\right)\right)\right]^{\lambda}, }
\end{aligned}
$$

with $\lambda=1 / \sqrt{2}$. Hence, all the hypotheses of Theorem 4 are satisfied. Furthermore, for some arbitrary $\mathfrak{a}_{1}, \ldots, \mathfrak{a}_{k} \in \mathcal{S}$, the sequence $\left\{\mathfrak{a}_{n}\right\}$ defined by (3) converges to $\mathfrak{a}^{*}=0$, which is the unique fixed point of mapping $\mathscr{L}$.

The upcoming result is an instant consequence of Theorem 4 by taking $\Theta(t)=e^{\sqrt{t}}$.

Corollary 1. Let $\mathscr{L}: \mathcal{S}^{k} \longrightarrow \mathcal{S}$ be a given mapping. Assume that there exists some $\lambda \in(0,1)$ such that

$$
\begin{gathered}
\sigma\left(\mathscr{L}\left(\mathfrak{a}_{1}, \ldots, \mathfrak{a}_{k}\right), \mathscr{L}\left(\mathfrak{a}_{2}, \ldots, \mathfrak{a}_{k+1}\right)\right) \\
\leq \lambda^{2} \max \left\{\sigma\left(\mathfrak{a}_{i}, \mathfrak{a}_{i+1}\right): \quad 1 \leq i \leq k\right\}
\end{gathered}
$$

for all $\left(\mathfrak{a}_{1}, \ldots, \mathfrak{a}_{k+1}\right) \in \mathcal{S}^{k+1}$ with $\mathscr{L}\left(\mathfrak{a}_{1}, \ldots, \mathfrak{a}_{k}\right) \neq \mathscr{L}$ $\left(\mathfrak{a}_{2}, \ldots, \mathfrak{a}_{k+1}\right)$. Then, for any arbitrary points $\mathfrak{a}_{1}, \ldots, \mathfrak{a}_{k} \in \mathcal{S}$, the sequence $\left\{\mathfrak{a}_{n}\right\}$ given by (3) converges to $\mathfrak{a}^{*}$, and $\mathfrak{a}^{*}$ is a fixed point of $\mathscr{L}$, that is, $\mathfrak{a}^{*}=\mathscr{L}\left(\mathfrak{a}^{*}, \ldots, \mathfrak{a}^{*}\right)$. Moreover, if

$$
\sigma(\mathscr{L}(\mathfrak{a}, \ldots, \mathfrak{a}), \mathscr{L}(\mathfrak{b}, \ldots, \mathfrak{b})) \leq \lambda^{2} \sigma(\mathfrak{a}, \mathfrak{b})
$$

holds for all $\mathfrak{a}, \mathfrak{b} \in \mathcal{S}$ with $\mathfrak{a} \neq \mathfrak{b}$, then $\mathfrak{a}^{*}$ is the unique fixed point of $\mathscr{L}$.

Corollary 2. Let $\mathscr{L}: \mathcal{S}^{k} \longrightarrow \mathcal{S}$ be a given mapping. Suppose that there exist $\lambda_{1}, \lambda_{2} \ldots, \lambda_{k}$ non-negative constants with $\lambda_{1}+$ $\lambda_{2}+\cdots+\lambda_{k}<1$ such that

$$
\begin{aligned}
& \sigma\left(\mathscr{L}\left(\mathfrak{a}_{1}, \ldots, \mathfrak{a}_{k}\right), \mathscr{L}\left(\mathfrak{a}_{2}, \ldots, \mathfrak{a}_{k+1}\right)\right) \\
& \leq \lambda_{1} \sigma\left(\mathfrak{a}_{1}, \mathfrak{a}_{2}\right)+\lambda_{2} \sigma\left(\mathfrak{a}_{2}, \mathfrak{a}_{3}\right) \\
& +\cdots+\lambda_{k} \sigma\left(\mathfrak{a}_{k}, \mathfrak{a}_{k+1}\right),
\end{aligned}
$$

for all $\left(\mathfrak{a}_{1}, \ldots, \mathfrak{a}_{k+1}\right) \in \mathcal{S}^{k+1} \quad$ with $\quad \mathscr{L}\left(\mathfrak{a}_{1}, \ldots, \mathfrak{a}_{k}\right)$ $\neq \mathscr{L}\left(\mathfrak{a}_{2}, \ldots, \mathfrak{a}_{k+1}\right)$. Then, for any arbitrary points $\mathfrak{a}_{1}, \ldots, \mathfrak{a}_{k} \in \mathcal{S}$, the sequence $\left\{\mathfrak{a}_{n}\right\}$ given by (3) converges to $\mathfrak{a}^{*}$, where $\mathfrak{a}^{*}$ is the unique fixed point of $\mathscr{L}$.

Proof. Evidently, (16) $\Longrightarrow$ (15) with $\lambda^{2}=\lambda_{1}+\lambda_{2}+\cdots+\lambda_{k}$. Now, let $\mathfrak{a}, \mathfrak{b} \in \mathcal{S}$ with $\mathfrak{a} \neq \mathfrak{b}$. From (16), we have

$$
\begin{aligned}
& \sigma(\mathscr{L}(\mathfrak{a}, \mathfrak{a}, \ldots, \mathfrak{a}), \mathscr{L}(\mathfrak{b}, \mathfrak{b}, \ldots, \mathfrak{b})) \\
& \leq \sigma(\mathscr{L}(\mathfrak{a}, \ldots, \mathfrak{a}), \mathscr{L}(\mathfrak{a}, \ldots, \mathfrak{a}, \mathfrak{b})) \\
& +\sigma(\mathscr{L}(\mathfrak{a}, \ldots, \mathfrak{a}, \mathfrak{b}), \mathscr{L}(\mathfrak{a}, \ldots, \mathfrak{a}, \mathfrak{b}, \mathfrak{b})) \\
& +\cdots+\sigma(\mathscr{L}(\mathfrak{a}, \mathfrak{b}, \ldots, \mathfrak{b}), \mathscr{L}(\mathfrak{b}, \mathfrak{b}, \ldots, \mathfrak{b})) \\
& \leq\left(\lambda_{k}+\lambda_{k-1}+\cdots+\lambda_{1}\right) \sigma(\mathfrak{a}, \mathfrak{b}) \\
& =\lambda^{2} \sigma(\mathfrak{a}, \mathfrak{b}),
\end{aligned}
$$

where $\lambda^{2}=\lambda_{k}+\lambda_{k-1}+\cdots+\lambda_{1} \in(0,1)$. Hence, all the hypotheses of Corollary 1 are satisfied.

Now consider the family $\Omega$ which contains large class of functions. For example, if

$$
\Theta(t)=2-\frac{2}{\pi} \arctan \left(\frac{1}{t^{\beta}}\right),
$$

where $0<\beta<1$ and $t>0$, we can obtain the following theorem from our main theorem.

Theorem 5. Let $\mathscr{L}: \mathcal{S}^{k} \longrightarrow \mathcal{S}$ be a given mapping. If there exist a mapping $\Theta \in \Omega$ and a constant $\beta, \lambda \in(0,1)$ such that

$$
\begin{aligned}
& 2-\frac{2}{\pi} \operatorname{arctanfrac1}\left(\sigma\left(\mathscr{L}\left(\mathfrak{a}_{1}, \ldots, \mathfrak{a}_{k}\right), \mathscr{L}\left(\mathfrak{a}_{2}, \ldots, \mathfrak{a}_{k+1}\right)\right)^{\beta}\right)
\end{aligned}
$$

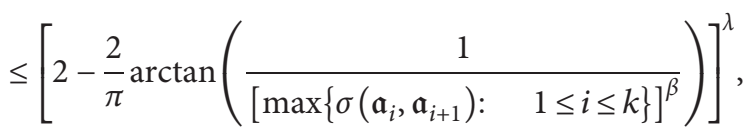

for all $\left(\mathfrak{a}_{1}, \ldots, \mathfrak{a}_{k+1}\right) \in \mathcal{S}^{k+1}$ with $\mathscr{L}\left(\mathfrak{a}_{1}, \ldots, \mathfrak{a}_{k}\right) \neq \mathscr{L}$ $\left(\mathfrak{a}_{2}, \ldots, \mathfrak{a}_{k+1}\right)$, then for any arbitrary points $\mathfrak{a}_{1}, \ldots, \mathfrak{a}_{k} \in \mathcal{S}$, the sequence $\left\{\mathfrak{a}_{n}\right\}$ given by (3) converges to $\mathfrak{a}^{*}$, and $\mathfrak{a}^{*}$ is a fixed point of $\mathscr{L}$, that is, $\mathfrak{a}^{*}=\mathscr{L}\left(\mathfrak{a}^{*}, \ldots, \mathfrak{a}^{*}\right)$. Moreover, if 


$$
\begin{aligned}
2-\frac{2}{\pi} \arctan & \frac{1}{\left(\sigma(\mathscr{L}(\mathfrak{a}, \ldots, \mathfrak{a}), \mathscr{L}(\mathfrak{b}, \ldots, \mathfrak{b}))^{\beta}\right)} \\
& \leq\left[2-\frac{2}{\pi} \arctan \left(\frac{1}{(\sigma(\mathfrak{a}, \mathfrak{b}))^{\beta}}\right)\right]^{\lambda},
\end{aligned}
$$

holds $\forall \mathfrak{a}, \mathfrak{b} \in \mathcal{S}$ with $\mathfrak{a} \neq \mathfrak{b}$, then $\mathfrak{a}^{*}$ is the unique fixed point of $\mathscr{L}$.

(1) Theorem 1.3 in [3] and Theorem 1.2 in [2] are direct generalizations of Theorem 4.

(2) Corollary 1 of Samet et al. in [11] can be deduced by putting $k=1$ in Theorem 4.

(3) Banach contraction principle [1] can be deduced from Corollaries 1 and 2 by taking $k=1$.

\section{Applications}

We start this section with the definition of equilibrium point as follows.

Definition 6. Let $\mathscr{L}: \mathcal{S}^{k} \longrightarrow \mathcal{S}$. For given $\mathfrak{a}_{1}$, $\mathfrak{a}_{2}, \ldots, \mathfrak{a}_{k} \in \mathcal{S}$, consider the recursive sequence $\left\{\mathfrak{a}_{n}\right\} \subset \mathcal{S}$ defined by

$$
\mathfrak{a}_{n+k}=\mathscr{L}\left(\mathfrak{a}_{n}, \mathfrak{a}_{n+1}, \ldots, \mathfrak{a}_{n+k-1}\right),
$$

for all $n \in \mathbb{N}$. A point $\overline{\mathfrak{a}}$ is said to be an equilibrium point of equation (17) if the following condition is satisfied:

$$
\overline{\mathfrak{a}}=\mathscr{L}(\overline{\mathfrak{a}}, \ldots, \overline{\mathfrak{a}})
$$

Definition 7. An equilibrium point $\overline{\mathfrak{a}}$ is said to be global attractor if for all $\mathfrak{a}_{1}, \mathfrak{a}_{2}, \ldots, \mathfrak{a}_{k} \in \mathcal{S}$, we have $\sigma\left(\mathfrak{a}_{n}, \overline{\mathfrak{a}}\right) \longrightarrow 0$ as $n \longrightarrow \infty$. by

If we have the recursive sequence $\left\{\mathcal{S}_{n}\right\} \subset P(N)$ defined

$$
\mathcal{S}_{n+k}=Q+\frac{1}{k} \sum_{i=0}^{k-1} E^{*} \psi\left(\mathcal{S}_{n+i}\right) E,
$$

for all $n \in \mathbb{N}$, then we explore the global attractivity of (59) in this application. In this application, we explore the global attractivity of (18). Here $P(N)$ (for $N \geq 2$ ) denotes family of $N \times N$ Hermitian positive definite matrices, $Q$ is an $N \times N$ Hermitian positive semidefinite matrix, $E$ is an $N \times N$ nonsingular matrix, $E^{*}$ is the conjugate transpose of $E$, and $\psi$ is a function from $P(N)$ to $P(N)$.

We first analyze the Thompson metric $\sigma$ on $P(N)$, which is defined by

$$
\sigma\left(E_{1}, E_{2}\right)=\max \left\{\log M\left(\frac{E_{1}}{E_{2}}\right), \log M\left(\frac{E_{2}}{E_{1}}\right)\right\},
$$

for $E_{1}, E_{2} \in P(N)$, where $M\left(E_{1} / E_{2}\right)=\inf \left\{\delta>0: \quad E_{1}\right.$ $\left.\leq \delta E_{2}\right\}=\delta^{+}\left(E_{2}^{-1 / 2} E_{1}^{-1 / 2} E_{2}\right)$, the maximal eigenvalue of $E_{2}^{-1 / 2} E_{1}^{-1 / 2} E_{2}$. Here, $E_{1} \leq E_{2}$ means that $E_{2}-E_{1}$ is positive semidefinite and $E_{1}<E_{2}$ means that $E_{2}-E_{1}$ is positive definite. Nussbaum [21] proved that $P(N)$ is a complete metric space regarding $\sigma$ and

$$
\sigma\left(E_{1}, E_{2}\right)=\left\|\ln \left(E_{1}^{-1 / 2} E_{2} E_{1}^{-1 / 2}\right)\right\|
$$

where $\|\cdot\|$ is a spectral norm $[21,22]$. Now we directly initiate the graceful properties of $\sigma$, i.e.,

$$
\sigma\left(E_{1}, E_{2}\right)=\sigma\left(E_{1}^{-1}, E_{2}^{-1}\right)=\sigma\left(M^{*} E_{1} M, M^{*} E_{2} M\right)
$$

for any nonsingular matrix $M$. The second valuable result is the nonpositive curvature property of $\sigma$ in this way.

$$
\sigma\left(E_{1}^{r}, E_{2}^{r}\right) \leq c \sigma\left(E_{1}, E_{2}\right), \quad c \in[0,1] .
$$

In accordance with (62) and (63), we get

$$
\sigma\left(M^{*} X^{r} M, M^{*} Y^{r} M\right) \leq|c| \sigma(X, Y), \quad c \in[-1,1],
$$

for all $E_{1}, E_{2} \in P(N)$.

Lemma 1 (see [23]). For all $E_{1}, E_{2}, E_{3}, E_{4} \in P(N)$, we have

$$
\sigma\left(E_{1}+E_{2}, E_{3}+E_{4}\right) \leq \max \left\{\sigma\left(E_{1}, E_{3}\right), \sigma\left(E_{2}, E_{4}\right)\right\} .
$$

Moreover, for all positive semidefinite $E_{1}$ and $E_{2}, E_{3} \in P(N)$, we have

$$
\sigma\left(E_{1}+E_{2}, E_{1}+E_{3}\right) \leq \sigma\left(E_{2}, E_{3}\right) .
$$

Let $\psi: P(N) \longrightarrow P(N)$ be a $\Theta$-contraction regarding $\sigma$. For $X_{1}, X_{2}, \ldots, X_{k} \in P(N)$, consider $\left\{X_{n}\right\} \subset P(N)$ defined by (59).

Theorem 8. Equation (59) has a unique equilibrium point $\bar{X} \in P(N)$. Furthermore, $\bar{X}$ is global attractor.

Proof. Define $\mathscr{L}: P(N)^{k} \longrightarrow P(N)$ by

$$
\begin{aligned}
& \mathscr{L}\left(M_{1}, M_{2}, \ldots, M_{k}\right) \\
& =Q+\frac{1}{k}\left[E^{*} \psi\left(M_{1}\right) E+E^{*} \psi\left(M_{2}\right) E+\cdots+E^{*} \psi\left(M_{k}\right) E\right],
\end{aligned}
$$

for all $M_{1}, M_{2}, \ldots, M_{k} \in P(N)$.

Let $M_{1}, M_{2}, \ldots, M_{k+1} \in P(N)$. By Lemma 1 , we get 


$$
\begin{aligned}
& \sigma \mathscr{L}\left(M_{1}, M_{2}, \ldots, M_{k}\right), \mathscr{L}\left(M_{2}, M_{3}, \ldots, M_{k+1}\right) \\
& =\sigma\left(Q+\frac{1}{k} \sum_{i=1}^{k} E^{*} \psi\left(M_{i}\right) E, Q+\frac{1}{k} \sum_{j=2}^{k+1} E^{*} \psi\left(M_{j}\right) E\right) \\
& \leq \sigma\left(\frac{1}{k} \sum_{i=1}^{k} E^{*} \psi\left(M_{i}\right) E, \frac{1}{k} \sum_{j=2}^{k+1} E^{*} \psi\left(M_{j}\right) E\right) \\
& =\sigma\left(\sum_{i=1}^{k}((1 / \sqrt{k}) E)^{*} \psi\left(M_{i}\right)((1 / \sqrt{k}) E), \sum_{j=2}^{k+1}((1 / \sqrt{k}) E)^{*} \psi\left(M_{j}\right)((1 / \sqrt{k}) E)\right) .
\end{aligned}
$$
have

Denote $O=(1 / \sqrt{k}) E$. Then, using again Lemma 1, we

$$
\begin{aligned}
& \sigma\left(\mathscr{L}\left(M_{1}, M_{2}, \ldots, M_{k}\right), \mathscr{L}\left(M_{2}, M_{3}, \ldots, M_{k+1}\right)\right) \\
& \leq \sigma\left(\sum_{i=1}^{k} O^{*} \psi\left(M_{i}\right) O, \sum_{j=2}^{k+1} O^{*} \psi\left(M_{j}\right) O\right) \\
& =\sigma\left(\begin{array}{c}
O^{*} \psi\left(M_{1}\right) O+O^{*} \psi\left(M_{2}\right) O+\cdots+ \\
O^{*} \psi\left(M_{k}\right) O, O^{*} \psi\left(M_{2}\right) O+ \\
O^{*} \psi\left(M_{3}\right) O+\cdots+O^{*} \psi\left(M_{k+1}\right) O
\end{array}\right) \\
& \leq \max \left\{\begin{array}{c}
\sigma\left(O^{*} \psi\left(M_{1}\right) O, O^{*} \psi\left(M_{2}\right) O\right), \\
\sigma\left(O^{*} \psi\left(M_{2}\right) O, O^{*} \psi\left(M_{3}\right) O\right), \ldots, \\
\sigma\left(O^{*} \psi\left(M_{k}\right) O, O^{*} \psi\left(M_{k+1}\right) O\right)
\end{array}\right\} \\
& =\max \left\{\sigma\left(O^{*} \psi\left(M_{j}\right) O, O^{*} \psi\left(M_{j+1}\right) O\right)\right\},
\end{aligned}
$$

for $j=1,2, \ldots, k$. As $A$ is nonsingular, $O$ is also nonsingular. By (19), $\forall j=1,2, \ldots, k$, we get

$$
\sigma\left(O^{*} \psi\left(M_{i}\right) O, O^{*} \psi\left(M_{i+1}\right) O\right)=\sigma\left(\psi\left(M_{i}\right), \psi\left(M_{i+1}\right)\right) .
$$
have

Now as $\psi$ is a $\Theta$-contraction, for all $j=1,2, \ldots, k$, we

$$
\Theta\left(\sigma\left(O^{*} \psi\left(M_{i}\right) O, O^{*} \psi\left(M_{i+1}\right) O\right)\right) \leq\left[\Theta\left(\sigma\left(M_{i}, M_{i+1}\right)\right)\right]^{\lambda},
$$

for some $\lambda \in(0,1)$. Thus, we have

$$
\begin{array}{r}
\Theta\left(\sigma\left(\mathscr{L}\left(M_{1}, M_{2}, \ldots, M_{k}\right), \mathscr{L}\left(M_{2}, M_{3}, \ldots, M_{k+1}\right)\right)\right) \\
\leq\left[\Theta\left(\max \left\{\sigma\left(M_{i}, M_{i+1}\right): \quad i=1,2, \ldots, k\right\}\right)\right]^{\lambda},
\end{array}
$$

for all $M_{1}, M_{2}, \ldots, M_{k+1} \in P(N)$. Thus, by Theorem 4 , there exists a global attractor equilibrium point $\bar{X} \in P(N)$.

Now for $M_{1}, M_{2} \in P(N)$ such that $\mathscr{L}\left(M_{1}\right.$, $\left.M_{1}, \ldots, M_{1}\right) \neq \mathscr{L}\left(M_{2}, M_{2}, \ldots, M_{2}\right)$, we have

$$
\begin{aligned}
& \Theta\left(\sigma\left(\mathscr{L}\left(M_{1}, M_{1}, \ldots, M_{1}\right), \mathscr{L}\left(M_{2}, M_{2}, \ldots, M_{2}\right)\right)\right) \\
& =\Theta\left(\sigma\left(Q+E^{*} \psi\left(M_{1}\right) E, Q+E^{*} \varphi\left(M_{2}\right) E\right)\right) \\
& \leq \Theta\left(\sigma\left(E^{*} \psi\left(M_{1}\right) E, E^{*} \psi\left(M_{2}\right) E\right)\right) \\
& =\Theta\left(\sigma\left(\psi\left(M_{1}\right), \varphi\left(M_{2}\right)\right)\right) \\
& \leq\left[\Theta\left(\sigma\left(M_{1}, M_{2}\right)\right)\right]^{\lambda} .
\end{aligned}
$$

Again, by Theorem 4, we get the unique equilibrium point.

\section{Conclusion}

Jleli and Samet [11] very recently exploited the idea of $\Theta$-contraction and established some generalized theorems for these contractions in generalized metric spaces. We continued their investigations and defined Prešić type $\Theta$-contraction. In this paper, we studied the convergence of iterative sequences of the Prešić type involving new classes of operators satisfying Prešić type $\Theta$-contractive condition in the context of metric spaces. Some examples are also provided to show the significance of the investigation of finding fixed points. As application, we derived some convergence results for a class of matrix difference equations.

\section{Data Availability}

The data supporting the findings of this study are included within the article.

\section{Conflicts of Interest}

The authors declare that they have no conflicts of interest.

\section{Acknowledgments}

The first author expresses his thanks and gratitude to King Abdulaziz University for unlimited support. 


\section{References}

[1] S. Banach, "Sur les opérations dans les ensembles abstraits et leur application aux équations intégrales," Fundamenta Mathematicae, vol. 3, pp. 133-181, 1922.

[2] S. B. Prešić, "Sur une classe d'inéquations aux différences finies et sur la convergence de certaines suites," Publications de l'Institut Mathématique, vol. 5, pp. 75-78, 1965.

[3] L. B. Ćirić and S. B. Prešić, "On Prešić type generalization of the Banach contraction mapping principle," Acta Mathematica Universitatis Comenianae, vol. 76, pp. 143-147, 2007.

[4] M. Păcurar, "A multi-step iterative method for approximating fixed points of Prešić-Kannan operators," Acta Mathematica Universitatis Comenianae, vol. 79, pp. 77-88, 2010.

[5] M. Abbas, M. Berzig, T. Nazir, and E. Karapınar, "Iterative approximation of fixed points for Prešić type $F$-contraction operators," UPB Scientific Bulletin, Series A, vol. 78, pp. 1-14, 2016.

[6] M. Pwfâcurar, "Approximating common fixed points of Presic-Kannan type operators by a multi-step iterative method," Analele Universitatii "Ovidius" Constanta-Seria Matematica, vol. 17, pp. 153-168, 2009.

[7] S. Shukla, "Prešić type results in 2-banach spaces," Afrika Matematika, vol. 25, no. 4, pp. 1043-1051, 2014.

[8] S. Shukla and R. Sen, "Set-valued Prešić-Reich type mappings in metric spaces," Revista de la Real Academia de Ciencias Exactas, Fisicas y Naturales. Serie A. Matematicas, vol. 108, no. 2, pp. 431-440, 2012.

[9] M. Berzig and B. Samet, "Solving systems of nonlinear matrix equations involving Lipshitzian mappings," Fixed Point Theory Application, vol. 2011, 89 pages, 2011.

[10] Y. Z. A. Chen, "Prešić type contractive condition and its applications," Nonlinar Analysis, vol. 71, pp. 2012-2017, 2009.

[11] M. Jleli and B. Samet, "A new generalization of the Banach contraction principle," Journal of Inequalities and Applications, vol. 2014, Article ID 38, 2014.

[12] A. Al-Rawashdeh and J. Ahmad, "Common fixed point theorems for JS-contractions," Bulletin of Mathematical Analysis and Applications, vol. 8, pp. 12-22, 2016.

[13] H. H. Al-Sulami and N. Hussain, J. Ahmad, Some generalized fixed point results with applications to dynamic programming," Journal of Function Spaces, vol. 2020, Article ID 8130764, 8 pages, 2020.

[14] A. Hussain, K. Tanzeela, Z. D. Mitrović, and S. Radenović, "Optimal solutions and applications to nonlinear matrix and integral equations via simulation function," Filomat, vol. 32, no. 12 , pp. 6087-6106, 2018.

[15] N. Hussain, J. Ahmad, L. Ćirić, and A. Azam, "Coincidence point theorems for generalized contractions with application to integral equations," Fixed Point Theory and Application, vol. 2015, Article ID 78, 2015.

[16] N. Hussain, J. Ahmad, and A. Azam, "On Suzuki-Wardowski type fixed point theorems," Journal of Nonlinear Sciences and Applications, vol. 8, no. 6, pp. 1095-1111, 2015.

[17] N. Hussain, J. Ahmad, and M. A. Kutbi, "Fixed point theorems for generalized Mizoguchi-Takahashi graphic contractions," Journal of Function Spaces, vol. 2016, Article ID 6514920, 7 pages, 2016.

[18] X. Li, A. Hussain, M. Adeel, and E. Savas, "Fixed point theorems for $Z_{e}$-contraction and applications to nonlinear integral equations," IEEE Access, vol. 7, pp. 120023-120029, 2019.

[19] Z. Ma, A. Hussain, M. Adeel, N. Hussain, and E. Savas, "Best proximity point theorems for generalized $\Theta$-contraction and application matrix equations," Symmetry, vol. 11, no. 1, p. 93, 2019, https://www.mdpi.com/2073-8994/11/1/93.

[20] F. Vetro, "A generalization of Nadler fixed point theorem," Carpathian Journal of Mathematics, vol. 31, no. 3, pp. 403410, 2015.

[21] R Nussbaum, "Hilbert's projective metric and iterated nonlinear maps," Memoirs of the American Mathematical Society, vol. 75, pp. 1-137, 1988.

[22] A. C. Thompson, "On certain contraction mappings in a partially ordered vector space," Proceedings of the American Mathematical Society, vol. 14, no. 3, p. 438, 1963.

[23] Y. Lim, "Solving the nonlinear matrix equation $X=Q+\sum_{i=1}^{m} A_{i}^{*} X^{\delta_{i}} A$ via a contraction principle," Linear Algebra and its Applications, vol. 430, pp. 1380-1383, 2009. 